\title{
Flavonoid combinations cause synergistic inhibition of proinflammatory mediator secretion from lipopolysaccharide-induced RAW 264.7 cells.
}

\begin{abstract}
Objectives: We evaluated several flavonoid combinations for synergy in the inhibition of proinflammatory mediator synthesis in the RAW 264.7 cellular model of inflammation. Methods: The inhibitory effect of chrysin, kaempferol, morin, silibinin, quercetin, diosmin and hesperidin upon nitric oxide (NO), prostaglandin E2 (PGE2) and tumour necrosis factor$\alpha(\mathrm{TNF}-\alpha)$ secretion from the LPS-induced RAW 264.7 monocytic macrophage was assessed and IC50 values obtained. Flavonoids that showed reasonable inhibitory effects in at least two out of the three assays were combined in a series of fixed IC50 ratios and reassessed for inhibition of NO, PGE2 and TNF- $\alpha$. Dose-response curves were generated and interactions were analysed using isobolographic analysis. Results: The experiments showed that only chrysin, kaempferol, morin, and silibinin were potent enough to produce dose-response effects upon at least two out of the three mediators assayed. Combinations of these four flavonoids showed that several combinations afforded highly significant synergistic effects. Conclusions: Some flavonoids are synergistic in their anti-inflammatory effects when combined. In particular chrysin and kaempferol significantly synergised in their inhibitory effect upon NO, PGE2 and TNF- $\alpha$ secretion. These findings open further avenues of research into combinatorial therapeutics of inflammatory-related diseases and the pharmacology of flavonoid synergy.
\end{abstract}

Keyword: Flavonoid; Isobologram; Nitric oxide; Prostaglandin E 2; RAW 264.7; Synergy; Tumour necrosis factor- $\alpha$. 